includes a database concerning patients, infrastructure and personnel, as a unique management tool. The GIS-based system enables to identify the location and current status of patients and providers at all times. During large-scale fires that occurred in Israel between November 22-27, 2016, which necessitated mass evacuation of populations, the information system was used to locate vulnerable patients and plan provision of needed services.

Results: Following the decree of mass evacuation of all populations from the risk zones due to the fires, the information system enabled the HMO to locate all vulnerable patients within minutes, and plan provision of specifically needed services: 2 patients from a nursing home and 1 home-care ventilated patient were located and evacuated within 2 hours. Specific medications were supplied within two hours to patients who were evacuated to absorption centers or hotels, based on their personal files available through the information system. One terminally ill patient was tracked and treated by the homecare unit within 3 hours, based on the data provided by the information system.

Conclusion: The comprehensive information system facilitated decision-making and improved ability of primary health care workers, to provide efficient and continuous medical care in the community during the disaster. During the recent fires in Israel, vulnerable patients were located within minutes and provided with individually-needed medical care within 2-3 hours, due to the availability of the information system that provided vital data concerning each patient.

Prehosp Disaster Med 2017;32(Suppl. 1):s71-s72

doi:10.1017/S1049023X17001911

The Introduction of Hospital MIMMS, A United Kingdom Based Hospital Mass Casualty Response Course to

Australia: Needs, Issues and Solutions

Peter Logan ${ }^{1}$, Carissa $\mathrm{Ob}^{2}$, Andrew Pearce ${ }^{3}$

1. National Hmimms Co-lead, National MIMMS Working Group, Brisbane/QLD/Australia

2. National Hmimms Co-lead, National MIMMS Working Group, Sydney/NSW/Australia

3. Chair, National MIMMS Working Group, Adelaide/Australia

Study/Objective: An outline of the introduction of a standardised, national, mass-casualty response course to Australian hospitals, including review of obstacles and issues experienced together with adopted solutions.

Background: Although the Advanced Life Support Group (ALSG) Major Incident Medical Management and Support Course (MIMMS) has been well established in Australia for several years, there was no corresponding, nationally consistent course providing hospital care providers with a similar framework for response. Several jurisdictions utilized locally applicable education, but an overall consistent national approach was absent.

Methods: Since a nationally consistent approach to hospitalbased mass-casualty response had been identified as desirable by the National MIMMS Working Group (NMWG), efforts were made to identify an appropriate, credible, and internationally recognized course. Funding was sought from the National Critical Care \& Trauma Response Center (NCCTRC), and negotiations with the UK based Advanced Life Support group ensued. In October 2014, two UK-based instructors traveled to Australia to provide an introductory course, and to train an initial cadre of Australian instructors with subsequent local roll out.

Results: The course was subjected to a process of iterative improvement based on participant feedback and instructor review. Many initial perceived issues revolved around definitions and terminology, which differed between the two nations. Significant attention was paid to the requirement for a course with a national remit, but which remained sufficiently flexible to adapt to the varying systems, processes, and procedures of the various Australian jurisdictions. HMIMMS has subsequently been adopted by four of six participating Australian jurisdictions, with 18 courses being run nationally between October 2014 and October 2016.

Conclusion: HMIMMS was successfully adapted to the Australian context, and adopted by four of the six participating jurisdictions. The remaining two may adopt HMIMMS at a later date. Australian implementation of HMIMMS demonstrates a successful collaboration of jurisdictions within a federated system. Prehosp Disaster Med 2017;32(Suppl. 1):s72

doi:10.1017/S1049023X17001923

\section{Maintaining Continuity of Care in the Recovery Phase}

With Family Medicine

Sneha Chacko, $M d^{1}$, Ritu R. Sarin ${ }^{1}$, Amalia Voskanyan ${ }^{1}$, Michael S. Molloy ${ }^{2}$, Gregory R. Ciottone ${ }^{1}$

1. The Bidmc Fellowship In Disaster Medicine, Department of Emergency Medicine BIDMC, Boston/MA/United States of America

2. Department Of Emergency Medicine, BIDMC Fellowship in Disaster Medicine, Boston/MA/United States of America

Study/Objective: The recovery period of a disaster often requires re-establishing health care and maintaining continuity of care for a large number of victims. This means recruiting primary care physicians who are preferably trained to provide healthcare to all ages, as well as having the ability to provide treatment covering a wide range of specialties. The specialty of Family Medicine is well-positioned to lead the healthcare recovery phase of disasters, prevent breach of medical care, manage chronic care issues, and provide urgent care treatment, thus alleviating the burden on the local emergency departments. Background: The medical recovery phase of a disaster is a chaotic period where re-establishing health care, and reconnecting the affected population to their primary care physicians becomes a complex challenge. This is partially due to displacement of the affected population, including the local physician force, as well as destruction of local clinics, both which are a necessity in resuming healthcare to full capacity.

Methods: Analysis of the recovery periods of multiple disasters over the past decade, and in different geographical locations for loss of primary care capacity.

Results: Deficit of primary care providers during the recovery period, breach in continuity of care for many patients, and lack of clinic guidelines were all identified in varying degrees in each disaster examined.

Conclusion: Activating specialists in Family Medicine to assume the lead during the disaster recovery phase will preserve 
continuity of primary care for many, as well as strengthen and accelerate the re-establishment of healthcare in the postdisaster period.

Prehosp Disaster Med 2017;32(Suppl. 1):s72-s73

doi:10.1017/S1049023X17001935

\section{The Role of Hospital Volunteers in Disaster Planning and Response \\ Alexandra Bernard}

Volunteer Services, McGill University Health Centre, Montreal/Canada

Study/Objective: Discuss hospital volunteers in disaster planning and response in a large, urban tertiary care center.

Background: Volunteer Services at The McGill University Health Centre (MUHC) began participating in disaster response and training following the 1989 École Polytechnique massacre in Montreal, Canada. Over time the program has grown and is now comprised of over 80 volunteers across two hospitals.

Methods: The MUHC volunteers were deployed in 2 official emergency situations (Concordia University massacre in 1992 \& Dawson College shooting in 2006), and provided support during the January 1998 North American ice storm. In addition, they participate in hospital emergency planning meetings, yearly disaster response training including live mass casualty simulations, and fan-out testing drills. The MUHC Volunteer Department has provided 10 to 80 simulated patients for each of the training exercises conducted since 2012. Results: Through participation in disaster planning and response, hospital volunteers were found to be a reliable, well trained, independent, un-costly and flexible source of manpower, familiar with the hospital environment and functioning. Specific roles have been identified and integrated into the external disaster response plan:

- Assist ED Nursing and Security teams, directing patients to designated disaster triage entrance

- Assist ambulatory patients to treatment areas after ED triage

- Perform regular ED Duties with an expected increased workload

- Participate in the Psychosocial Response with other members of the multidisciplinary Psychosocial Response Team

- Perform regular inventory of disaster response materials Frequent turnover and limited funds for training are factors limiting further involvement of hospital volunteers in emergency management.

Conclusion: Hospital volunteers are a valuable asset to the hospital during an external disaster. Their involvement in emergency management activities including regular attendance at planning meetings and participation in simulations are key to successful collaboration when external disasters occur.

Prehosp Disaster Med. 2017;32(Suppl. 1):s73

doi:10.1017/S1049023X17001947

\author{
Catastrophic Data Disruptions: A New Frontier for \\ Disaster Preparedness \\ Puneet Seth \\ Emergency Medicine, Singhealth (Sengkang Hospital), Singapore/ \\ Singapore
}

Study/Objective: To outline the causes, effects, and mitigation strategies of catastrophic health care data disruption.

Background: Recent years have seen a surge in disruption of health care delivery due to various causes. Health care data has become a target for a variety of actors, especially in the field of cyberspace. As we become more urbanized, we have increasingly become dependent on information technology to retain and retrieve long and complicated patient medical records to allow us to practice medicine safely and efficiently. Thus, the cyber-physical system has become a key infrastructure item that needs to be protected against threats.

Methods: A detailed Internet search was conducted to look for possible causes, effects, and mitigation strategies of catastrophic data disruption. The findings were listed in the results.

Results: Risks to Patient Data Systems emanate from: 1. Targeted cyber-attacks:

- Disgruntled employees;

- Cybercriminals- especially for Ransomware;

- Nation states.

2. Business risk to any part of the data chain:

- Attack on database provider's cloud;

- Financial bankruptcy of any part of the chain (vendor/ sub-vendor);

- Physical infrastructural damage to servers/storage systems.

3. Power outage and related IT failure. 4. Solar flares. 5. EMP weapons. Immediate effects on health care delivery are: 1. Cascading failure of health delivery. 2. No medical records can be retrieved, bringing care to a halt. 3. Medications cannot be served safely. 4 . Impaired care of patients on critical care support - ICUs, EDs, hemodialysis, and chemotherapy units. 5. No refilling of prescriptions. Best Practices in Operations Continuity involve: 1 . Crisis Communications protocol for a confirmed outage. 2. Scenario-based preparedness exercises: Downtime Protocol. 3. Easy-to-deploy paper-based recording material: Downtime toolkit. 4. Service recovery with alternative offsite hardware and software providers. 5. Cloud-based backup redundancy arrangements.

Conclusion: Catastrophic Data Disruptions are a new frontier in disaster preparedness. It involves everyone and can be crippling if not prepared for in advance.

Prehosp Disaster Med. 2017;32(Suppl. 1):s73

doi:10.1017/S1049023X17001959

Ensuring Operational Continuity of Community Healthcare Services During Disasters

Gal Horowitz

Medical Quality Control, Emergency And Disaster Management, kupat holim meuhedet, Tel- Aviv Yafo/Israel

Study/Objective: To present contribution of a national guideline on the capacity of community primary care health care systems to establish and sustain operational continuity during disasters.

Background: "Meuhedet" is the $3^{\text {rd }}$ largest Health Maintenance Organization (HMO) in Israel, insuring $15 \%$ of the population ( 1,200,000 members). The HMO is committed to 